\title{
Cover story \\ Getting into the red: a strategic step for safety
}

\section{IN THE BEGINNING...}

In 1993 we examined the first 2000 incident reports using the Australian Incident Monitoring Study (AIMS) and tried to formulate ways of reducing untoward events. One of the major recommendations was aimed at avoiding drug errors. There were 144 incidents related to drugs. Of these, 58 incidents were syringe drug swaps and, of these, $71 \%$ involved muscle relaxants. This is a classic example of the manager's "80/20 rule" in which $80 \%$ of the adverse events will come from about $20 \%$ of the drugs. One of the recommended changes was the colour coding of syringes by the drug types they contained. In 1993 there were Standards for Drug Labelling in the $\mathrm{USA}^{2}{ }^{2}$ Canada, ${ }^{3}$ and South Africa. At the recommendation of the Australian Society of Anaesthetists, Standards Australia wrote an Australian/New Zealand Standard for the labelling of anaesthetic drugs. A standard for labelling of drugs was developed that used the same basic colour scheme as the three existing national standards. ${ }^{4}$ However, the Australasian standard also added requirements on the lettering design and on the quality of the label adhesive as both aspects had been identified as potential problems. Within this standard the relaxants were colour identified by a strong red. It was hoped that simply identifying the relaxant syringes could avoid about $70-80 \%$ of the syringe swap incidents.

\section{A FURTHER STEP..}

To lock in the relationship with red and relaxants, it was decided to try to get a red syringe produced. This, it was hoped, might reduce labelling errors and give a clear indication of the relaxant syringe from all angles. Although the projected sales of such a syringe were only in the

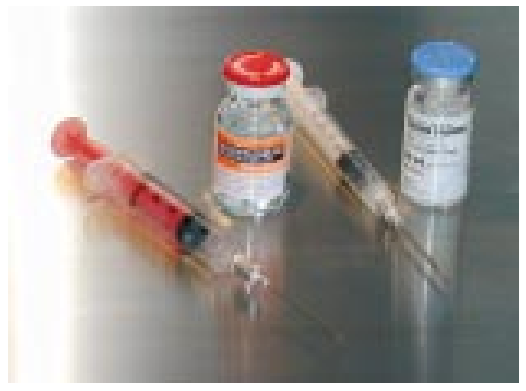

the red syringe with other drugs and then thinking that they had not given the relaxant! We have had few reports to date of problems in using the red syringe strategy. There is one report of an argument with one surgeon who liked the colour so much that he wanted to use a red syringe for his local anaesthetic block. We are now analysing 8000 incident reports and hope to report soon on the impact of our intervention.

The strategy of identifying the relaxants with the red plunger syringes depends on strictly using the syringe only for relaxants. This is a clear systems solution to a problem which, despite our best intentions, could not be resolved. Clearly identifying relaxant drugs may help to avoid the very distressing incidents of patients being paralysed because of a syringe swap before they are induced. In the interests of patient safety, every department should consider "getting into the red".

\section{ACKNOWLEDGEMENTS}

HAVE WE MADE A DIFFERENCE...?

This is a major question which the present AIMS incident collection is trying to answer. Initially the use of the red syringe exclusively for relaxants was not widely adopted, except in South Australia. Only about $10 \%$ of anaesthetists in Australia were involved and therefore the reduction in relaxant incidents could be expected to be small even if the change was completely effective. However, now that the red plunger syringe is used in all Australian states and the current usage has risen to about a quarter of a million annually, we are hopeful that a difference in the syringe swap error will soon be apparent.

The current results are encouraging. Anaesthetists readily identify the red syringe and, indeed, there have been some reports of anaesthetists refilling
Thanks to Ruth Kumar, MS, for her creative expertise in arranging and shooting the cover image.

\section{REFERENCES}

1 Currie M, Mackay P, Morgan C, et al. The "wrong drug" problem in anaesthesia: an wrong drug problem in anaesthesia: an
analysis of 2000 incident reports. Anaesthesia Intens Care 1993; 21: 596-601.

2 American Society for Testing and Materials. Standard specification for user applied drug labels in anesthesiology. American Society for Testing and Materials D4774-94, 1995.

3 Canadian Standards Association. Standard for user-applied drug labels in anaesthesia and critical care. Z264.3-98. Etobicoke, Canada: Canadian Standards Association, 1998

4 AS/NZS. User-applied labels for use on syringes containing drugs used during anaesthesia. AS/NZS 4375, 1996.

W John Russell Department of Anaesthesia \& Intensive Care, Royal Adelaide Hospital, Adelaide, South Australia 5000 